\title{
Vinorelbine plus trastuzumab combination as first-line therapy for HER 2-positive metastatic breast cancer patients: an international phase II trial
}

\author{
A Chan ${ }^{*, 1}$, M Martin ${ }^{2}$, M Untch ${ }^{3}$, MG Gil ${ }^{4}$, V Guillem-Porta ${ }^{5}$, M Wojtukiewicz ${ }^{6}$, P Kellokumpu-Lehtinen $^{7}$,

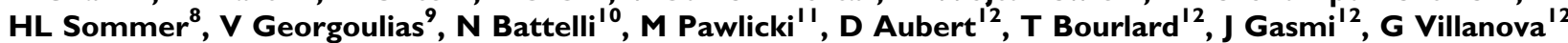 \\ and L Petruzelka ${ }^{13}$ on behalf of the Navelbine ${ }^{\circledR}$ Herceptin ${ }^{\circledR}$ Project \\ 'Mount Hospital and Royal Perth Hospital, Perth, Australia; ${ }^{2}$ Hospital Clinico San Carlos, Madrid, Spain; ${ }^{3}$ Klinikum Grosshadern, München, Germany; \\ ${ }^{4}$ Instituto Catala d'Oncologia, Hospitalet de Llobregat, Barcelona, Spain; ${ }^{5}$ Instituto Valenciano de Oncologia, Valencia, Spain; ${ }^{6}$ Oncology Department, \\ Medical University and Regional Cancer Centre, Bialystok, Poland; ${ }^{7}$ Medical School, Tampere University Central Hospital, Pikonlinna, Finland; ${ }^{8}$ Klinikum \\ Universität München, München, Germany; ${ }^{9}$ Peripheral General Hospital Iraklion, Stavrakia and Voutes, Iraklion, Greece; ${ }^{10}$ Azienda Ospedaliera Umberto \\ I, Ancona, Italy; " 'Centrum Onkologii W Krakowie, Krakow, Poland; ${ }^{2}$ Institut de Recherche Pierre Fabre, Boulogne-Billancourt, France; ${ }^{13}$ General Teaching \\ Hospital, Prague, Czech Republic
}

The aim of this international phase II trial was to determine the efficacy and safety profile of weekly vinorelbine plus trastuzumab as first-line chemotherapy for women with HER 2-overexpressing metastatic breast cancer. Sixty-nine patients with tumours overexpressing HER 2 received vinorelbine: $30 \mathrm{mg} \mathrm{m}^{-2}$ week $^{-1}$ and trastuzumab: $4 \mathrm{mg} \mathrm{kg}^{-1}$ on day I as a loading dose followed by $2 \mathrm{mg} \mathrm{kg}^{-1}$ week $^{-1}$ starting on day 8. Sixty-two patients were evaluable for response and 69 patients were evaluable for toxicity. The overall response rate was $62.9 \%$. The median time to response was 8.4 weeks, the median duration of response was 17.5 months, the median progression-free survival was 9.9 months $(95 \% \mathrm{Cl}, 5.6-12.1)$ and the one-year progression-free survival was $39.1 \%$. The median survival for all patients was 23.7 months $(95 \% \mathrm{Cl}$, 18.4-32.6). This regimen was safe: grade 3-4 neutropenia were observed over $17.7 \%$ of courses in $83.8 \%$ of patients, with only two episodes of febrile neutropenia $(0.1 \%)$ in two patients $(2.9 \%)$. Only one patient discontinued treatment due to grade 3 symptomatic cardiac dysfunction that resolved with therapy. Vinorelbine plus trastuzumab is one of the most active treatment regimens for patients with HER 2-positive metastatic breast cancer and demonstrates a very favourable safety profile allowing prolonged treatment with long-term survival. This study has been presented in part at the following conferences: The San Antonio Breast Cancer Symposium, San Antonio, TX, USA, 2003; The American Society of Clinical Oncology, Orlando, FL, USA, 2005.

British Journal of Cancer (2006) 95, 788-793. doi:I0.I038/sj.bjc.660335 I www.bjcancer.com

Published online 12 September 2006

(c) 2006 Cancer Research UK

Keywords: trastuzumab; vinorelbine; first-line chemotherapy; HER 2-positive metastatic breast cancer

Advances in understanding breast cancer biology have led to the development of targeted anticancer treatment. HER 2/neu gene amplification, identified in $20-30 \%$ of breast cancers, is a prognostic marker for poor clinical outcomes (Slamon et al, 1987, 1989; Berger et al, 1988; Ravdin and Chamness, 1995). Trastuzumab is a humanised monoclonal antibody that targets the extracellular domain of the HER 2 receptor and inhibits the proliferation of human tumour cells overexpressing the HER 2 protein. Trastuzumab has demonstrated antitumour activity in metastatic breast cancer in first-line treatment, or in patients who have tumour progression after chemotherapy (Cobleigh et al, 1999; Vogel et al, 2002). Preclinical studies indicated synergistic or

*Correspondence: Dr A Chan, Suite 4I, I 46 Mounts Bay Road, Perth 6000, Australia; E-mail: arlene.chan@bigpond.com

Received 25 May 2006; revised 31 July 2006; accepted I August 2006; published online 12 September 2006 additive interactions for trastuzumab with different chemotherapy agents (Pegram et al, 1999, 2004). Two randomised trials comparing trastuzumab plus chemotherapy to chemotherapy alone demonstrated an improved outcome for patients receiving trastuzumab plus chemotherapy (Slamon et al, 2001; Marty et al, 2005). However, trastuzumab therapy can be associated with cardiac toxicity (Slamon et al, 2001). Retrospective analyses have found that concomitant use with anthracyclines and advanced age are predictive factors of trastuzumab-induced cardiac dysfunction (Seidman et al, 2002).

Among chemotherapeutic agents used in the management of metastatic breast cancer, vinorelbine has demonstrated the greatest antitumour synergy with trastuzumab in preclinical models (Pegram et al, 1999, 2004). Furthermore, the weekly administration of both agents and the lack of overlapping toxicity between these drugs make this combination suitable for further investigation. Several single institution or national phase II trials have been 
conducted with this regimen as first-line therapy or in heavily pretreated patients with metastatic breast cancer. These studies consistently demonstrated high efficacy with limited toxicities (Burstein et al, 2001, 2003; Jahanzeb et al, 2002; Bayo-Calero et al, 2004; Bernardo et al, 2004).

This international, open-label, phase II study was designed to evaluate the efficacy and safety of this combination as first-line treatment for metastatic breast cancer.

\section{MATERIALS AND METHODS}

\section{Eligibility criteria}

Eligible patients were female $>18$ years, with histologically proven metastatic breast carcinoma. Other inclusion criteria included Karnofsky performance status (KPS) score $\geqslant 70 \%$, at least one bidimensionally measurable lesion according to the World Health Organisation (WHO) criteria and a life expectancy $>16$ weeks. Adjuvant chemotherapy containing an anthracycline was allowed if the total cumulative dose of doxorubicin or epirubicin did not exceed 360 and $720 \mathrm{mg} \mathrm{m}^{-2}$, respectively. A disease-free interval of more than 6 months between the last dose of adjuvant chemotherapy and documentation of relapse was needed. Patients were required to give written informed consent before studyspecific procedures were performed and to be able to comply with the protocol for the duration of the study. Patients were required to have normal cardiac function with left ventricular ejection fraction (LVEF) measured by echocardiography or multigated acquisition scan (MUGA scan) no more than $10 \%$ below the normal limit of the institution.

Patients were ineligible if they had local relapse only, prior chemotherapy in the metastatic setting, previous treatment with vinca alkaloid or trastuzumab, grade $>2$ peripheral neuropathy, serious illness or medical conditions such as cardiac disease, unstable diabetes, uncontrolled hypercalcaemia or active infection. Patients were also excluded if they were pregnant or lactating, had central nervous system or leptomeningeal metastases, had participated in another clinical trial with any investigational drug within 30 days prior to the study inclusion or had a history of another malignancy, except cured basal cell carcinoma of the skin or excised carcinoma in situ of the cervix.

Patients were required to have adequate bone marrow (haemoglobin value $\geqslant 10 \mathrm{~g} \mathrm{dl}^{-1}$, absolute neutrophil count $\geqslant 1.5 \times 10^{9} 1^{-1}$ and platelet count $\geqslant 100 \times 10^{9} 1^{-1}$ ), renal (serum creatinine $\leqslant 130 \mu \mathrm{moll}^{-1}$ ) and liver function (serum bilirubin $\leqslant 1.5 \times$ upper normal limit (UNL), serum glutamic-oxalic transaminase (SGOT) or serum glutamic-pyruvic transaminase $(\mathrm{SGPT}) \leqslant 2.5 \mathrm{UNL}$, unless liver involvement).

The HER 2 status of primary or metastatic tumours was assessed by immunohistochemistry (IHC) and fluorescent in situ hybridisation (FISH) on formalin-fixed paraffin-embedded tissue. Because of the possibility of discrepancy between local laboratories for the HER 2 testing with IHC, a centralised testing was implemented (Professor Josef Rüschoff, Institute of Pathology, Kassel, Germany). Immunohistochemistry was performed using the DAKO Hercept ${ }^{\circledR}$ Test, and FISH analysis was performed using the PathVysion HER 2 probe kit Vysis ${ }^{\circledR}$. Patients with HER 2 staining assessed $3+$ by IHC were eligible for the study, while those with IHC $2+$ required confirmation by FISH.

\section{Treatment plan}

Treatment was given on a weekly basis ( 1 cycle $=4$ weeks of treatment). The initial trastuzumab infusion was administered intravenously at the dose of $4 \mathrm{mg} \mathrm{kg}^{-1}$ over $90 \mathrm{~min}$ on day 1 . The patient remained under medical supervision for $1 \mathrm{~h}$ following completion of the first infusion. Subsequent weekly trastuzumab courses were given at $2 \mathrm{mg} \mathrm{kg}^{-1}$ over $30 \mathrm{~min}$. The postinfusion observation period for the second infusion was shortened to $30 \mathrm{~min}$ and was withheld over subsequent administrations provided no adverse events occurred.

Vinorelbine was administered after trastuzumab infusion at $30 \mathrm{mg} \mathrm{m}^{-2}$ weekly over $6-10 \mathrm{~min}$. The first dose of vinorelbine was administered $2 \mathrm{~h}$ after the trastuzumab infusion. Patients were to receive at least 8 weeks of treatment (two cycles).

Diphenhydramine and paracetamol premedication were optional. Treatment was continued until disease progression, unacceptable toxicity or patient's refusal to continue.

\section{Dose modifications}

There was no dose adjustment for trastuzumab. The infusion was stopped if the patient developed chills, fever, allergic reaction or any grade 3-4 toxicities. Trastuzumab administration was also withheld in the event of a decline in LVEF value either $\geqslant 20 \%$ of absolute units from baseline to a value above the lower normal limit for the centre, or $\geqslant 10 \%$ of absolute units to a value below the lower normal limit for the centre. Trastuzumab therapy was reintroduced 1 month later if there was no further decline in LVEF. Trastuzumab was permanently interrupted if New York Heart Association (NYHA) class III/IV cardiac function impairment occurred.

Vinorelbine was postponed for 1 week in case of grade $\geqslant 3$ neutropenia. Following one delay of vinorelbine, the dose of vinorelbine was permanently decreased to $25 \mathrm{mg} \mathrm{m}^{-2}$ week $^{-1}$ for the remainder of study treatment. In the event of grade $>2$ peripheral neuropathy, vinorelbine was permanently discontinued. In case of increased SGOT/SGPT to 5.1-20.0 UNL, or bilirubin at 1.5-3.0 UNL, vinorelbine administration was postponed for 1 week and permanently discontinued in case of three consecutive delays or SGOT/SGPT value $>20.0 \mathrm{UNL}$ and/or total bilirubin value $>3$ UNL. Patients who experienced grade 3 or 4 neutropenia with or without fever were allowed to receive a granulocyte colonystimulating factor in the subsequent cycles at the investigator's discretion.

\section{Study assessments}

Screening assessments were performed at baseline in all patients within 28 days before study treatment, including medical history, physical examination, performance status, HER 2 testing, electrocardiogram, LVEF, chest X-ray, tumour measurements by computed tomography (CT), abdominal ultrasound, bone scan and brain CT scan. On day 1 of each 4-week cycle, physical examination and performance status were assessed and haematological and blood chemistry measurements were carried out. Additional radiological imaging was performed as clinically indicated.

Complete blood cells counts were performed weekly within 2 days before vinorelbine administration. LVEF measurement was repeated every 6 months and at any time if clinically indicated.

Objective responses were assessed every 8 weeks until progression, or less than 8 weeks if early progression was suspected. The best overall response achieved, using the standard WHO criteria of tumour response, was reported for each patient. A complete response (CR) required complete disappearance of all previously detectable disease with no appearance of new lesions, and partial response (PR) required at least a $50 \%$ decrease of the sum of the products of the two greatest perpendicular diameters of all measurable lesions, with no appearance of new lesions or progression of any lesion. Both $\mathrm{CR}$ and $\mathrm{PR}$ had to be confirmed 4 weeks later. Stable disease (SD) was defined as less than $50 \%$ decrease or a less than $25 \%$ increase in the size of one or more measurable lesions. Progressive disease was defined as $25 \%$ or more increase of previously described lesions, or appearance of 
new lesions. An Independent Review Panel confirmed all responses. Clinical benefit was defined as patients achieving CR, PR or SD maintained for a minimum of 6 months.

Adverse events and medical history were recorded throughout the study. The WHO criteria were used to grade the adverse events.

\section{Statistical analysis}

The one-sample multiple testing procedure for phase II clinical trials described by Fleming was used. Based on a type I error rate of $5 \%$ and a $95 \%$ power to reject the null hypothesis of a $20 \%$ objective response rate (complete or partial), a sample size of 60 evaluable patients was needed.

All registered patients were included in the intent-to-treat (ITT) analysis; patients who received at least one administration of study treatment were analysed for safety. The objective response was the main end point for this study. The Kaplan-Meier method was applied on overall survival, progression-free survival, duration of response and time to treatment failure.

\section{RESULTS}

\section{Patients' characteristics}

Between October 2000 and June 2002, 69 patients with metastatic breast cancer screened for HER 2 status and fulfilling the eligibility criteria were enrolled in the study at 20 sites from 13 countries. Seven out of 69 patients were not evaluable for response, but were included in the ITT analysis: two patients were not assessable as a result of premature study discontinuation (one patient withdrew from the study after the first therapy administration due to an adverse event, the second one died during the first cycle from sepsis), one patient had bone metastases only which was not assessable, another patient had no measurable disease as defined in the protocol and three other patients were not eligible due to relapse within 6 months from early-stage chemotherapy. Therefore, 62 patients with measurable disease were assessable for disease response as per protocol.

The patient's characteristics are described in Table 1. Median age was 53 years (range 30-74 years), more than $70 \%$ of patients had KPS $\geqslant 90 \%$ and the median time between initial diagnosis and relapse was 17.8 months. Nearly all patients $(97.1 \%)$ had tumour HER 2 status $3+$ determined by IHC, while two patients required confirmation with FISH. The majority of patients had visceral metastasis (75.4\%). Sixty-five per cent of patients had prior neo/ adjuvant chemotherapy including anthracycline in the majority of cases.

\section{Clinical efficacy}

Independent review panel observed an objective response rate of $62.9 \%$ among the 62 evaluable patients (95\% CI, 49.7-74.8), including $14.5 \%$ of CRs. Stable disease was observed in $19.4 \%$ of patients. The clinical benefit $(\mathrm{CR}+\mathrm{PR}+\mathrm{SD} \geqslant 6$ months) was $72.6 \%$ (95\% CI, 61.5-83.7) Table 2. In the ITT population, the objective response rate was of 58\% (95\% CI, 45.5-69.8).

Patients who had received prior adjuvant chemotherapy with anthracyclines or anthracyclines and taxanes had similar objective response rates (61.9 and 54.5\%, respectively), as well as those with or without visceral involvement (63.8 and $60 \%$ of objective response rates, respectively) Table 2 . Patients anticipated to have a worse prognosis, with a disease-free interval of less than 12 months, had an overall objective response rate of $71.4 \%$. The median follow-up of patients was 36.2 months. The median time to response was 8.4 weeks ( $7.1-31.3$ weeks) and the median duration of response was 17.5 months (95\% CI, 12.1-23.0). The time to treatment failure was 6 months (95\% CI, 5.3-8.6), the progressionfree survival was 9.9 months (95\% CI, 5.6-12.1) and the median
Table I Patient's characteristics

\begin{tabular}{|c|c|c|}
\hline Characteristic & $\mathbf{N}$ & (\%) \\
\hline Included & 69 & \\
\hline \multicolumn{3}{|l|}{ Age (years) } \\
\hline Median & 53 & \\
\hline Range & $30-74$ & \\
\hline$K P S \geqslant 70 \%$ & 69 & 100 \\
\hline \multicolumn{3}{|l|}{ HER 2 testing } \\
\hline $\mathrm{IHC} 3+$ & 67 & 97.1 \\
\hline $\mathrm{IHC} 2+/ \mathrm{FISH}+$ & 2 & 2.9 \\
\hline Prior hormonotherapy & 34 & 49.3 \\
\hline Prior chemotherapy & 45 & 65.2 \\
\hline Neoadjuvant & 5 & 7.2 \\
\hline Adjuvant & 30 & 43.5 \\
\hline Both & 10 & 14.5 \\
\hline \multicolumn{3}{|l|}{ Chemotherapy regimen } \\
\hline Anthracycline based & 23 & 51.1 \\
\hline Anthracycline+taxane & 13 & 28.9 \\
\hline CMF & 9 & 20.0 \\
\hline Metastatic sites $\geqslant 2$ & 22 & 31.9 \\
\hline Lymph nodes & 35 & 50.7 \\
\hline Visceral (lung/liver) & 52 & 75.4 \\
\hline Liver & 38 & 55.1 \\
\hline Lung & 20 & 29.0 \\
\hline Bone & 31 & 44.9 \\
\hline \multicolumn{3}{|c|}{ Disease-free interval from diagnosis to first relapse (months) } \\
\hline Median & 17.8 & \\
\hline Range & $0-202.7$ & \\
\hline
\end{tabular}

$\mathrm{CMF}=$ cyclophosphamide methotrexate 5 -fluorouracil; FISH = fluorescent in situ hybridisation; $I \mathrm{HC}=$ immunohistochemistry; $\mathrm{KPS}=$ Karnofsky performance status.

Table 2 Antitumour efficacy

Best overall response (Independent Review Panel) $\mathbf{N}$

\begin{tabular}{|c|c|}
\hline Evaluable patients & 62 \\
\hline CR & 914.5 \\
\hline PR & 3048.4 \\
\hline Objective response $(\mathrm{CR}+\mathrm{PR})(95 \% \mathrm{Cl})$ & $3962.9(49.7-74.8)$ \\
\hline SD & 1219.4 \\
\hline Clinical benefit (CR+PR+SD $\geqslant 6$ months) $(95 \% \mathrm{Cl})$ & $4572.6(61.5-83.7)$ \\
\hline PD & 1117.7 \\
\hline \multicolumn{2}{|l|}{ Prognostic factors of response rate (patients) } \\
\hline \multicolumn{2}{|l|}{ Prior chemotherapy regimen } \\
\hline No prior adjuvant/neoadjuvant (23) & 1669.6 \\
\hline Anthracycline based $(2 \mathrm{I})$ & 1361.9 \\
\hline Anthracycline+taxane (II) & 654.5 \\
\hline \multicolumn{2}{|l|}{ Visceral involvement (liver/lung) } \\
\hline Yes (47) & 3063.8 \\
\hline No $(15)$ & 960.0 \\
\hline Stage IV at diagnosis (12) & 975.0 \\
\hline
\end{tabular}

$\mathrm{Cl}=$ confidence interval; $\mathrm{CR}=$ complete response; $\mathrm{PD}=$ progressive disease; $\mathrm{PR}=$ partial response; $\mathrm{SD}=$ stable disease.

overall survival for all patients was 23.7 months (95\% CI, 18.432.6) (Table 3; Figures 1 and 2).

Twenty-six patients received further radiotherapy, 24 patients received hormonotherapy and 39 patients received further chemotherapy. 
Table 3 Survival data (cutoff date I October, 2004)

\begin{tabular}{lc}
\hline Median time to response (weeks) (range) & $8.4(7.1-31.3)$ \\
Median duration of response (months) $(95 \% \mathrm{Cl})$ & $17.5(12.1-23.0)$ \\
Median progression-free survival (months) $(95 \% \mathrm{Cl})$ & $9.9(5.6-12.1)$ \\
Median survival (months) $(95 \% \mathrm{Cl})$ & $23.7(18.4-32.6)$ \\
\hline
\end{tabular}

$\mathrm{Cl}=$ confidence interval

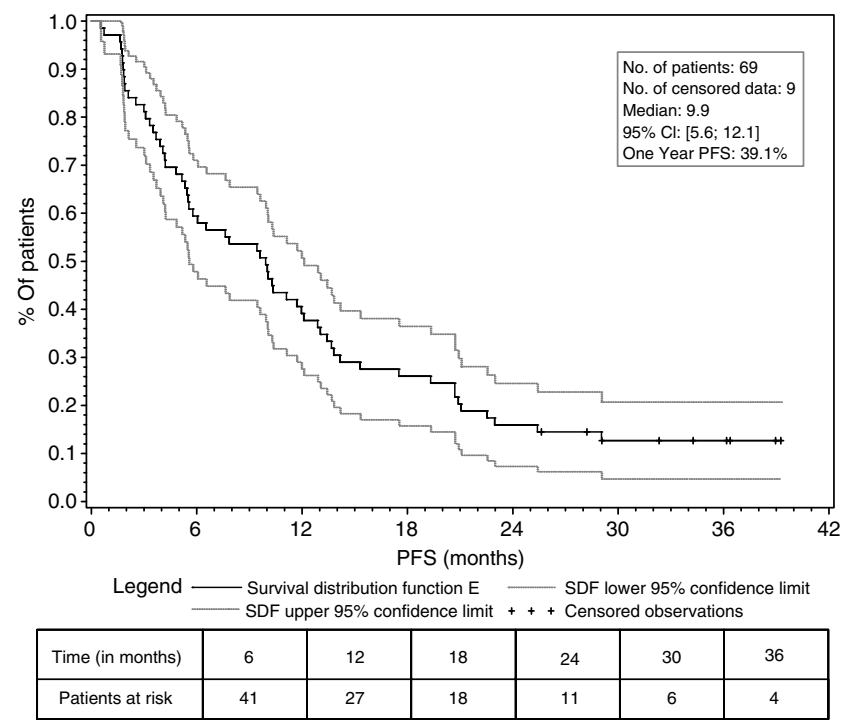

Figure I Progression-free survival - ITT population.

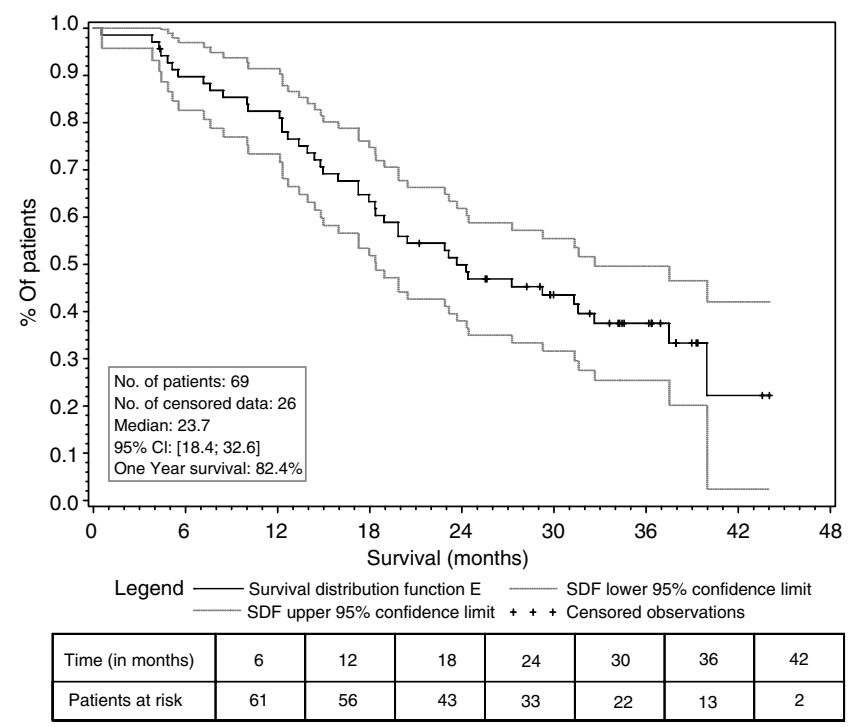

Figure 2 Overall survival - ITT population.

\section{Dose modifications/reductions}

A total of 1769 administrations of vinorelbine and 2242 administrations of trastuzumab were given, with a median number of 18 (range, 1-106) and 24 (range, 1-124) administrations per patient for vinorelbine and trastuzumab, respectively. Fourteen patients received this regimen for more than 1 year, and four patients for more than 2 years.

The median relative dose intensity for vinorelbine was $66.1 \%$, whereas that for trastuzumab was $95 \%$. Vinorelbine treatment was delayed more than 3 days or cancelled in $26.5 \%$ of administrations mainly due to haematological toxicity, whereas trastuzumab treatment was delayed more than 3 days or cancelled in $6.7 \%$ of administrations mainly due to investigator's decision or patient's convenience.

\section{Treatment-related toxicity}

The most frequent haematological toxicity (WHO grading) was neutropenia, with grade 3-4 neutropenia observed in $83.8 \%$ of patients over $301(17.7 \%)$ administrations. Neutropenia was usually brief, infrequently associated with infections and was not cumulative (data not shown). There were only two episodes $(0.1 \%)$ of febrile neutropenia in two patients $(2.9 \%)$. One of these patients died from neutropenic sepsis. Grade 3 anaemia was reported in only two $(2.9 \%)$ patients over three $(0.2 \%)$ courses. No grade 2,3 or 4 thrombocytopenia was reported (Table 4).

Nonhaematological toxicities were mild. The most common nonhaematological toxicity (WHO grade 3/4) attributed to study treatment was grade 3 asthenia in six patients $(8.8 \%)$ and infection in four patients (5.9\%). Other side effects were rare: grade 3 peripheral neuropathy and constipation were noticed in two patients each $(2.9 \%)$ (Table 5).

\section{Cardiac toxicity}

Eligible patients received study treatment with LVEF no more than $10 \%$ below the normal limit of the institution. The mean LVEF at baseline was $63.8 \%$ (95\% CI, 62.1-65.6), and the minimum value during treatment remained similar at a mean of $59.1 \%(95 \% \mathrm{CI}$, 55.9-61.7) (Figure 3).

One patient experienced a decline of LVEF to $38 \%$ with symptomatic cardiac failure 4 months after study initiation. She was aged 68 years old with a history of hypertension. Vinorelbine administration was postponed for 2 weeks and trastuzumab treatment was permanently discontinued. Following initiation of antifailure treatment, the symptoms resolved with the LVEF normalising at $58 \%$. Three other patients experienced asymptomatic decline in LVEF to 41,46 and $44 \%$, respectively. The therapy was interrupted for 4 weeks and only reintroduced after LVEF increased to $\geqslant 50 \%$.

\section{DISCUSSION}

Trastuzumab combined with chemotherapy is the standard of care for women with HER 2-overexpressing metastatic breast cancer. However, the optimal chemotherapeutic agent to combine with trastuzumab is not established. Ideally, chemotherapy-trastuzumab combinations should produce durable response rates with minimal toxicity. In particular, the known cardiac toxicity of trastuzumab would preclude its use with an agent also known to be cardiotoxic. Data from preclinical models have demonstrated synergistic interaction between trastuzumab and vinorelbine (Pegram et al, 2004). Several phase II studies have been conducted in the first-line setting or after progression to other chemotherapy. This doublet has produced consistent response rates ranging from 60 to $86 \%$, with a satisfactory tolerance profile (Burstein et al, 2001, 2003; Jahanzeb et al, 2002; Bernardo et al, 2004; Bayo-Calero et al, 2004).

The current study evaluated the combination of vinorelbine $\left(30 \mathrm{mg} \mathrm{m}^{-2}\right.$ week $\left.^{-1}\right)$ and trastuzumab $\left(4 \mathrm{mg} \mathrm{kg}^{-1}\right.$ on day 1 followed by $2 \mathrm{mg} \mathrm{kg}^{-1}$ week $^{-1}$ starting on day 8) as first-line therapy for HER 2-positive metastatic breast cancer patients. This is the first international phase II trial with centralised HER 2 testing evaluating this combination.

The short disease-free interval of 17.8 months and prior use of adjuvant anthracyclines with or without taxane chemotherapy in $52.2 \%$ of patients underlies the aggressive nature of disease in this 
Table 4 Worst haematological WHO grades, by evaluable patient $\left(N=68^{\mathrm{a}}\right)$ and by evaluable administration of vinorelbine+trastuzumab $(\mathrm{N}=1705)$

\begin{tabular}{|c|c|c|c|c|c|}
\hline \multirow[b]{2}{*}{ Haematological toxicity } & \multirow[b]{2}{*}{ Patients (overall incidence) } & \multicolumn{2}{|c|}{ Patients } & \multicolumn{2}{|c|}{ Administrations } \\
\hline & & Grade 3, N (\%) & Grade 4, $N(\%)$ & Grade 3, $N(\%)$ & Grade 4, $N$ (\%) \\
\hline Leucopenia & $63(92.6)$ & $39(57.4)$ & $6(8.8)$ & $159(9.3)$ & $13(0.8)$ \\
\hline Neutropenia & $63(92.6)$ & $28(41.2)$ & $29(42.6)$ & $226(13.3)$ & $75(4.4)$ \\
\hline Febrile neutropenia $^{b}$ & $2(2.9)$ & - & - & - & - \\
\hline Thrombocytopenia & $4(5.9)$ & - & - & - & - \\
\hline \multicolumn{6}{|c|}{$\begin{array}{l}\text { WHO = World Health Organisation. }{ }^{a} \text { One patient with only one treatment administration was not evaluable for haematological toxicity. }{ }^{b} \text { One of these patients died of } \\
\text { neutropenic sepsis. }\end{array}$} \\
\hline \multicolumn{6}{|c|}{ Table 5 Worst related nonhaematological WHO grades, by evaluable patient $(N=69)$ and by evaluable cycle $(N=6 \mid 3)$ of vinorelbine+trastuzumab } \\
\hline & & & ents & & \\
\hline Nonhaematological toxicity & Patients (overall incidence) & Grade 3, N (\%) & Grade 4, N (\%) & Grade 3, N (\%) & Grade $4, N$ (\%) \\
\hline Diarrhoea & $18(26.1)$ & I (1.4) & I ( 1.4$)$ & I $(0.2)$ & I $(0.2)$ \\
\hline Stomatitis & $27(39.1)$ & - & - & - & - \\
\hline Peripheral neuropathy & $22(31.9)$ & $2(2.9)$ & - & $3(0.5)$ & - \\
\hline Asthenia & $33(47.8)$ & $6(8.7)$ & - & $10(1.6)$ & - \\
\hline Chills & $15(21.7)$ & I (1.4) & - & I (0.2) & - \\
\hline Hair loss & $25(36.2)$ & - & - & - & - \\
\hline Cutaneous & $14(20.3)$ & I (1.4) & - & I (0.2) & - \\
\hline Drug fever & $23(33.3)$ & I (1.4) & - & I $(0.2)$ & - \\
\hline Infection & $18(26.1)$ & $4(5.8)$ & - & $5(0.9)$ & - \\
\hline Local venous toxicity & $16(23.2)$ & I (1.4) & - & $2(0.3)$ & - \\
\hline
\end{tabular}

$\mathrm{WHO}=$ World Health Organisation

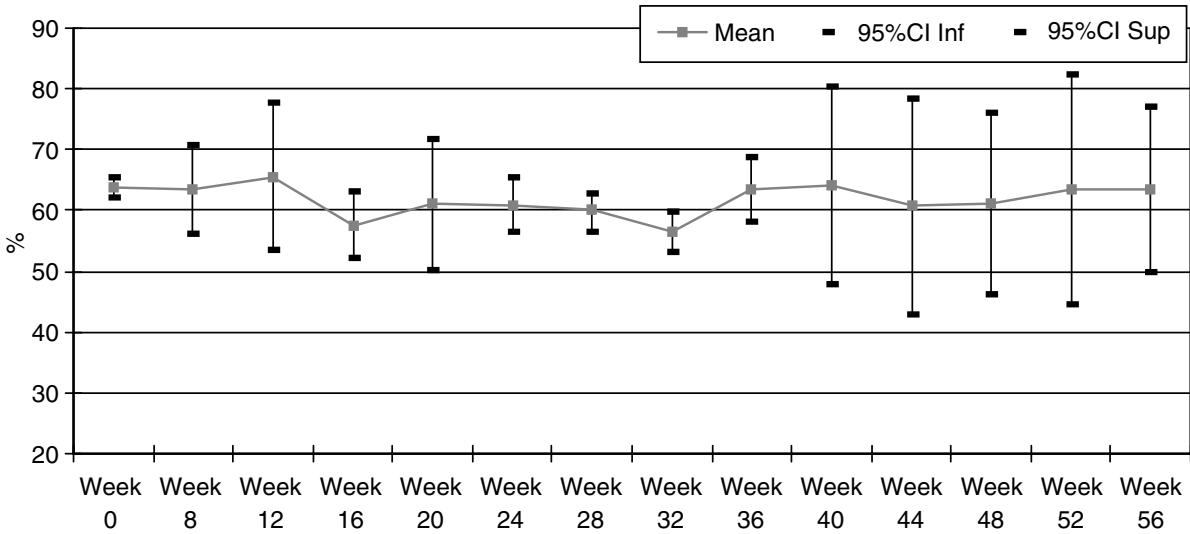

Figure 3 Mean LVEF during treatment.

population under study. The clinical benefit of $72.6 \%$ with a median duration of response of 17.5 months compares favourably with other trastuzumab combinations (Seidman et al, 2001; Esteva et al, 2002; Marty et al, 2005). The duration of efficacy of the current combination is reflected by the $39.1 \%$ disease-free survival rate at 1 year. By 9 November 2005, two patients were still on treatment with vinorelbine and trastuzumab after 3.5-3.6 years, respectively.

This multicentre study confirms the efficacy of vinorelbine with trastuzumab previously seen in other studies, with independent radiological review avoiding investigator assessment bias. Burstein reported $75 \%$ of response rates without grade $3-4$ cardiac toxicity in a single centre trial of weekly vinorelbine $\left(25 \mathrm{mg} \mathrm{m}^{-2}\right)$ and trastuzumab $\left(4 \mathrm{mg} \mathrm{kg}^{-1}\right.$ on day 1 then $2 \mathrm{mg} \mathrm{kg}^{-1}$ week $\left.^{-1}\right)$ used as first- and second-line therapy (Burstein et al, 2001). In a multi- institutional trial of the same schedule, the same author confirmed the efficacy of trastuzumab plus vinorelbine as first-line chemotherapy, with $68 \%$ of patients achieving an objective response and $39 \%$ of patients remaining free of disease progression at 1 year (Burstein et al, 2003). Jahanzeb et al (2002), in a trial of first-line vinorelbine and trastuzumab at the same schedule and doses as the current study, reported $78 \%$ of responses among 40 patients. In addition, the time to disease progression was extended to 17 months.

The results are in line with other reports using different chemotherapies in combination with trastuzumab. The objective response rate was of $50 \%$ in the study by Slamon et al (2001) with the combination of trastuzumab and paclitaxel and $61 \%$ in the study by Marty et al (2005) with trastuzumab plus docetaxel. 
The toxicities associated with vinorelbine and trastuzumab were predictable and manageable. Although grade 3 or 4 neutropenia occurred in $83.8 \%$ of patients, only two episodes $(0.1 \%)$ of febrile neutropenia occurred ( $2.9 \%$ of patients).

Cardiomyopathy, a serious toxicity associated with trastuzumab-based therapy, has been reported in $13 \%$ of patients receiving paclitaxel and trastuzumab (including severe events in $2 \%$ of patients) (Slamon et al, 2001). In the study by Marty et al (2005), one patient (1\%) receiving trastuzumab and docetaxel experienced symptomatic heart failure, and another patient experienced symptomatic heart failure 5 months after discontinuation of trastuzumab while receiving an investigational anthracycline for 4 months. The favourable safety profile of the vinorelbine/trastuzumab combination has been observed in other trials: Burstein et al (2003) reported only one patient with grade 3 cardiac toxicity, whereas no grade 3-4 cardiac toxicity was seen by Jahanzeb et al (2002).

It is important to note that cardiac toxicity was reported in $4.7 \%$ of patients with trastuzumab monotherapy (Cobleigh et al, 1999). The $5.9 \%$ incidence of cardiac toxicity seen in the present study with only one patient $(1.5 \%)$ experiencing symptomatic cardiac failure is therefore similar to that seen when trastuzumab is used alone, reflecting the absence of additional cardiac toxicity from

\section{REFERENCES}

Bayo-Calero JL, Mayordomo-Camara JI, Sanchez-Rovira P, Perez-Carrion R, Illaramendi JJ, Garcia-Bueno JJ, Gonzalez-Flores E, Crespo C, RamosVazquez M, Garcia-Palomo A (2004) A multicenter study with trastuzumab and vinorelbine as first and second-line treatment in patients with HER 2-positive metastatic breast cancer. Breast Cancer Res Treat 88(Suppl 1): S207 (abstract 5069)

Berger MS, Locher GW, Saurer S, Gullick WJ, Waterfield MD, Groner B, Hynes NE (1988) Correlation of c-erbB-2 gene amplification and protein expression in human breast carcinoma with nodal status and nuclear grading. Cancer Res 48: $1238-1243$

Bernardo G, Palumbo R, Bernardo A, Villani G, Melazzini M, Poggi G, Frascaroli M, Jedrychowska I (2004) Final results of a phase II study of weekly trastuzumab and vinorelbine in chemonaive patients with HER 2overexpressing metastatic breast cancer. Proc Am Soc Clin Oncol 22: 59s (abstract 731)

Burstein HJ, Harris LN, Kelly Marcom P, Lambert-Falls R, Havlin K, Overmoyer B, Friedlander RJ, Gargiulo J, Strenger R, Vogel CL, Ryan PD, Ellis MJ, Nunes RA, Bunnel CA, Campos SM, Hallor R, Gelman R, Winer EP (2003) Trastuzumab and vinorelbine as first-line therapy for HER 2overexpressing metastatic breast cancer: multicenter phase II trial with clinical outcomes, analysis of serum tumor markers as predictive factors, and cardiac surveillance algorithm. J Clin Oncol 21: 2889-2895

Burstein HJ, Kuter I, Campos SM, Gelman RS, Tribou L, Parker LM, Manola J, Younger J, Matulonis U, Bunnell CA, Partridge AH, Richardson PG, Clarke K, Shulman LN, Winer EP (2001) Clinical activity of trastuzumab and vinorelbine in women with HER 2-overexpressing metastatic breast cancer. J Clin Oncol 19: 2722-2730

Cobleigh MA, Vogel CL, Tripathy D, Robert NJ, Scholl S, Fehrenbacher L, Wolter JM, Paton V, Shak S, Lieberman G, Slamon DJ (1999) Multinational study of the efficacy and safety of humanized anti-HER 2 monoclonal antibody in women who have HER 2-overexpressing metastatic breast cancer that has progressed after chemotherapy for metastatic disease. J Clin Oncol 17: 2639-2648

Esteva FJ, Valero V, Booser D, Guerra LT, Murray JL, Pusztai L, Cristofanilli M, Arun B, Esmaeli B, Fritsche HA, Sneige N, Smith TL, Hortobagyi GN (2002) Phase II study of weekly docetaxel and trastuzumab for patients with Her 2-overexpressing metastatic breast cancer. J Clin Oncol 20: $1800-1808$

Jahanzeb M, Mortimer JE, Yunus F, Irwin DH, Speyer J, Koletsky AJ, Klein P, Sabir T, Kronish L (2002) Phase II trial of weekly vinorelbine and trastuzumab as first-line therapy in patients with HER 2+ metastatic breast cancer. Oncologist 7: 410-417

Marty M, Cognetti F, Maraninchi D, Snyder R, Mauriac L, Tubiana-Hulin M, Chan S, Grimes D, Anton A, Lluch A, Kennedy J, O’Byrne K, Conte vinorelbine. Twenty per cent of patients were treated for more than 1 year.

In conclusion, the vinorelbine/trastuzumab regimen is an effective and well-tolerated first-line therapy option for HER 2overexpressing metastatic breast cancer patients. The shorter duration of administration without the need of a steroid premedication and lack of cumulative side effects with marginal cardiac toxicity provide strong argument for its choice as the control arm in future phase III trials including trastuzumab-based combinations.

\section{ACKNOWLEDGEMENTS}

We thank the following investigators of this trial: F Majois (Hopital Jolimont, Haine-Saint-Paul, Belgium), MO Bednarik (Masakikuv Oncologicky Ustav, Brno, Czech Republic), UR Kleeberg (Haematologisch-Onkologische Praxis Altona, Hamburg, Germany), K Wong (Medical Oncology Pte Ltd, Singapore), A Stewart (Christie Hospital, Manchester, UK), K Buser (Sonnenhof-Klinik Engeried, Bern, Switzerland) and G Westman (University Hospital, Malmö, Sweden). We are indebted to all the patients whose participation made this study possible, and to $\mathrm{P}$ Miquel and to $\mathrm{Y}$ Parlier for editorial assistance.
PF, Green M, Ward C, Mayne K, Extra JM (2005) Randomized phase II trial of the efficacy and safety of trastuzumab combined with docetaxel in patients with human epidermal growth factor receptor 2-positive metastatic breast cancer administered as first-line treatment: The M77001 Study Group. J Clin Oncol 23: 4265-4274

Pegram M, Hsu S, Lewis G, Pietras R, Beryt M, Sliwkowski M, Coombs D, Baly D, Kabbinawar F, Slamon D (1999) Inhibitory effects of combinations of HER-2/neu antibody and chemotherapeutics agents used for treatment of human breast cancer. Oncogene 18: 2241-2251

Pegram MD, Konecny GE, O'Callaghan C, Beryt M, Pietras R, Slamon DJ (2004) Rational combinations of trastuzumab with chemotherapeutic drugs used in the treatment of breast cancer. J Natl Cancer Inst 96: 739749

Ravdin PM, Chamness GC (1995) The c-erbB-2 proto-oncogene as a prognostic and predictive marker in breast cancer: a paradigm for the development of other macromolecular markers - a review. Gene 159: $19-27$

Seidman A, Hudis C, Pierri MK, Shak S, Paton V, Ashby M, Murphy M, Stewart SJ, Keefe D (2002) Cardiac dysfunction in the trastuzumab clinical trials experience. J Clin Oncol 20: 1215-1221

Seidman AD, Fornier MN, Esteva FJ, Tan L, Kaptain S, Bach A, Panageas KS, Arroyo C, Valero V, Currie V, Gilewski T, Theodoulou M, Moynahan ME, Moasser M, Sklarin N, Dickler M, D'Andrea G, Cristofanilli M, Rivera E, Hortobagyi GN, Norton L, Hudis CA (2001) Weekly trastuzumab and paclitaxel for metastatic breast cancer with analysis of efficacy by Her 2 immunophenotype and gene amplification. J Clin Oncol 19: 2587-2595

Slamon DJ, Clark GM, Wong SG, Levin WJ, Ullrich A, McGuire WL (1987) Human breast cancer: correlation of relapse and survival with amplification of the HER-2/neu oncogene. Science 235: 177-182

Slamon DJ, Godolphin W, Jones LA, Holt JA, Wong SG, Keith DE, Levin WJ, Stuart SG, Udove J, Ullrich A (1989) Studies of the HER-2/neu proto-oncogene in human breast and ovarian cancer. Science 244: $707-712$

Slamon DJ, Leyland-Jones B, Shak S, Fuchs H, Paton V, Bajamonte A, Fleming T, Eiermann W, Wolter J, Pegram M, Baselga J, Norton L (2001) Use of chemotherapy plus a monoclonal anti-body against HER 2 for metastatic breast cancer that overexpresses HER 2. N Engl J Med 344: $783-792$

Vogel CL, Cobleigh MA, Tripathy D, Gutheil JC, Harris LN, Fehrenbacher L, Slamon DJ, Murphy M, Novotny WF, Burchmore M, Shak S, Stewart SJ, Press M (2002) Efficacy and safety of trastuzumab as a single agent in first-line treatment of HER 2-overexpressing metastatic breast cancer. J Clin Oncol 20: 719-726 